\title{
Fabrication and characterization of collagen (shell) /thermoplastic polyurethane (core) composite nanofibers by coaxial electrospinning
}

\author{
Rui Chen, ${ }^{1 *}$ Xiao Qiang $\mathrm{Li}^{2}{ }^{2}$ Qin Fei Ke, ${ }^{1}$ Chuang Long He, ${ }^{3}$ Xiu Mei Mo ${ }^{3}$ \\ ${ }^{1 *}$ College of textiles, Donghua University, Shanghai, 201620, People's Republic of \\ China; tel: (+86) (0)21-67792741; fax: (+86) (0)21-67792653; email: chenrui@mail. \\ dhu.edu.cn. \\ ${ }^{2}$ College of materials science and technology, Donghua University, Shanghai, \\ 201620, People's Republic of China. \\ ${ }^{3^{*}}$ College of chemistry and chemical engineering and biological engineering, \\ Donghua University, Shanghai, 201620, People's Republic of China.
}

(Received: 20 December, 2008; published: 07 February, 2010)

\begin{abstract}
Coaxial-electrospinning is a new method used in tissue engineering and drug delivery field. It can spin nanofibers with shell-core structure through electrospinning of two polymer solutions. In this study, collagen was used as the outer layer or the shell and thermoplastic polyurethane (TPU) was used as the inner layers or the core. A series of tests were conducted to characterize the compound nanofiber and its membrane. Morphology and microscopy of the ultrafine fibers were characterized by scanning electron microscopy (SEM) and transmission electron microscopy (TEM), whereas the fiber diameter distribution was understood using image visualization software (ImageJ) and measurements of solution viscosity and conductivity. Mechanical measurements were carried out by applying tensile test loads to samples which were prepared from electrospun ultra fine non-woven fiber mats. The results demonstrated that these composite nanofibers had the characters of native extracellular matrix and they could be designed as the scaffold for tissue engineering and functional biomaterials.

Key words: coaxial electrospinning, collagen/thermoplastic polyurethane, mechanical performance, nanofiber
\end{abstract}

\section{Introduction}

The regeneration of damaged or lost tissue requires that certain function reparative cells assemble three-dimensionally around and inside the supporting scaffold via a series of biological activities such as adhering, migrating, growing and differentiating to attain a proper integration between cells and scaffold for synthesizing the new tissue [1] The technology of tissue engineering (TE) aims to generate new or substitute tissues for malfunctioning ones and could well become an alternative method to whole organ transplantation [2,3].

Most of these human organs deposited on fibrous structures with the fibril/fiber size realigning from nanometer to millimeter scale. So nanofiber has now been extensively used to mimic these natural tissue matrixes. At present, electrospinning is the most prevalent process that can create nanofibers through an electrically charged jet of polymer solution or polymer melt. Different processing parameters such as kind of polymer, viscosity, surface tension, jet charge density, temperature and humidity 
control the electrospinning process, especially the diameter and morphology of the resulting fibers [4].

Effective use of polymer nanofibrous scaffolds for tissue engineering relies not only on the construction of the fibers in nanoscales being able to mimic the physical structure of the native extracellular matrix, but also on the biochemistry characteristics of the materials used. One method of functionalizing nanofibers is realized by employing an advanced coaxial electrospinning technology. Through combination of different materials in the axial or radial direction, novel properties and functionalities for nanoscale devices can be anticipated. The unique core-shell structure offers a number of potential benefits.

As candidate materials, pure TPU and collagen have already been electrospun into nanofibers as biomaterials respectively $[5,6]$, and they all have biological benefits to be used as TE scaffolds. The promising study of electrospun TPU and collagen coaxial compound nanofiber has not been well understood. The primary objective of this study was to investigate the coaxial electrospinning to obtain the surface functionalized compound nanofibers.

\section{Results and discussion}

\section{Solvent selection}

In the electrospinning system, there are a number of parameters affecting fiber morphology and fiber diameter, such as polymer concentration/viscosity, applied voltage, needle diameter and the delivery rate of polymer solution [7]. Additionally, the solvent used to dissolve the polymer has a significant effect on the spinning effect on the spinnability of the polymer solution and fiber morphology. In this study, we used HFP as the solvent for the electrospinning process and fabrication of the fibrous scaffolds. HFP is an ideal organic solvent. It allows full extension of the polymer and it evaporates completely after the fiber formation process without leaving any residue on the formed fibers [8]. In the non-optimal conditions, we found that when we used the TPU (N,N-dimethylformamide or tetrahydrofuran as solvent) and collagen solution (HFP as solvent) in the process of co-electrospinning, there was white deposition at the bottom of the coaxial spinneret, and the experiment could not be carried through. On the other side, when we used HFP as solvent for both TPU and collagen, the Taylor cone at the bottom of the spinneret was clear and did not have any impurity or deposition. So we selected the HFP as a proper solvent for electrospinning of collagen-TPU blends.

\section{Morphology}

The polymers of TPU and collagen were dissolved in HFP separately. The two solutions were put into the coaxial spinneret. Through observing the structure of almost conical electrified menisci consisting of an outer meniscus surrounding an inner one, we successfully fabricated the shell-core composite nanofibers. The SEM photographs of pure TPU, collagen and TPU/collagen with core-shell structure composite nanofibers are shown in Fig. 1, whereas their nanofibers diameters distributions were further determined and the results are shown in Tab. 1.

Nanofiber diameters of each weight ratio were calculated from the diameter of 100 nanofibers which was directly measured from SEM photographs. From the fiber 
diameter distribution, we could found that TPU had the largest number of average diameter, while collagen had the smallest number of average diameter.
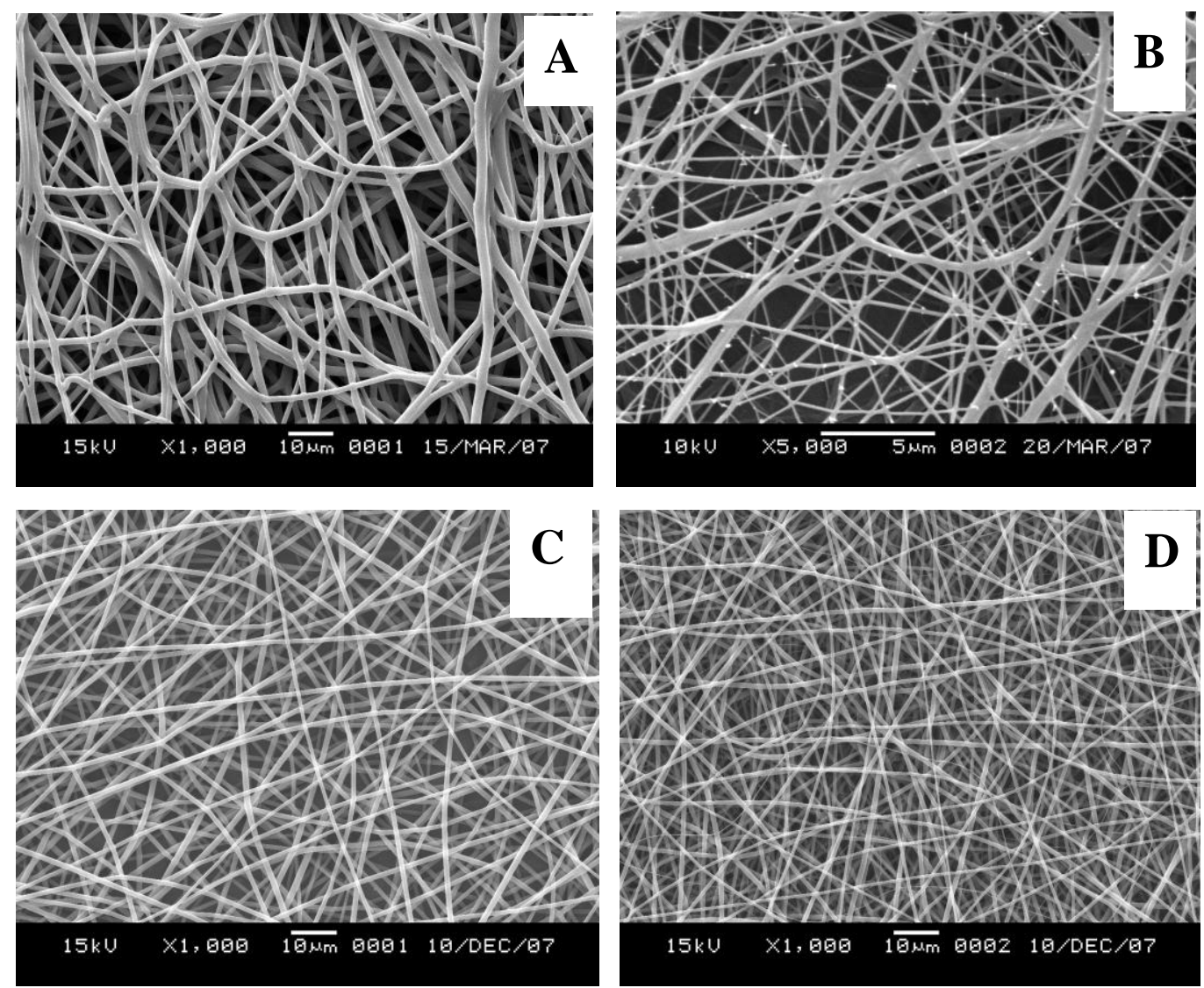

Fig. 1. SEM images of pure TPU, collagen and collagen/TPU coaxial electrospun nanofibers. (A) SEM image of pure TPU; (B) SEM image of pure collagen; (C) SEM image of coaxial electrospun nanofibers of collagen (shell)/TPU (core) (8 wt\%/3 wt\%); (D) SEM image of coaxial electrospun nanofibers of collagen (shell)/TPU (core) (8 wt $\% / 6 \mathrm{wt} \%)$

Fig. 2 gives the conductivity and the [ $\eta$ ] as functions of different spinning solutions. It was found that these different types of spinning solutions have different kind of solution properties. The conductivity slightly decreased, and conversely, [n] obviously increased with TPU content increasing in the spinning solution. However, the fiber diameter increased with increasing concentration TPU content and concentration of the complex solution. This indicated that the fiber diameter is mainly affected by the concentration of solution under the same electrospinning process diameter.

It has been known that in electrospinning, the force that causes the stretching of the solution is due to the repulsive forces between the charges on the electrospinning jet [9]. Collagen was typical amphiprotic macromolecule electrolyte. When collagen was affiliated, more ions were formed in the shell solution. So a higher discharge density could be carried by the electrospinning jet. On the other hand, the increased charge carried by the solution would increase the stretching of the solution. And the increased stretching of the solution also could tend to yield fibers of smaller diameter. In general, we found that the core concentration of TPU solution was between 3\% 
and $6 \%$ and could get smooth nanofibers. A lower or higher concentration would lead to significant beads on the surface of nanofibers.

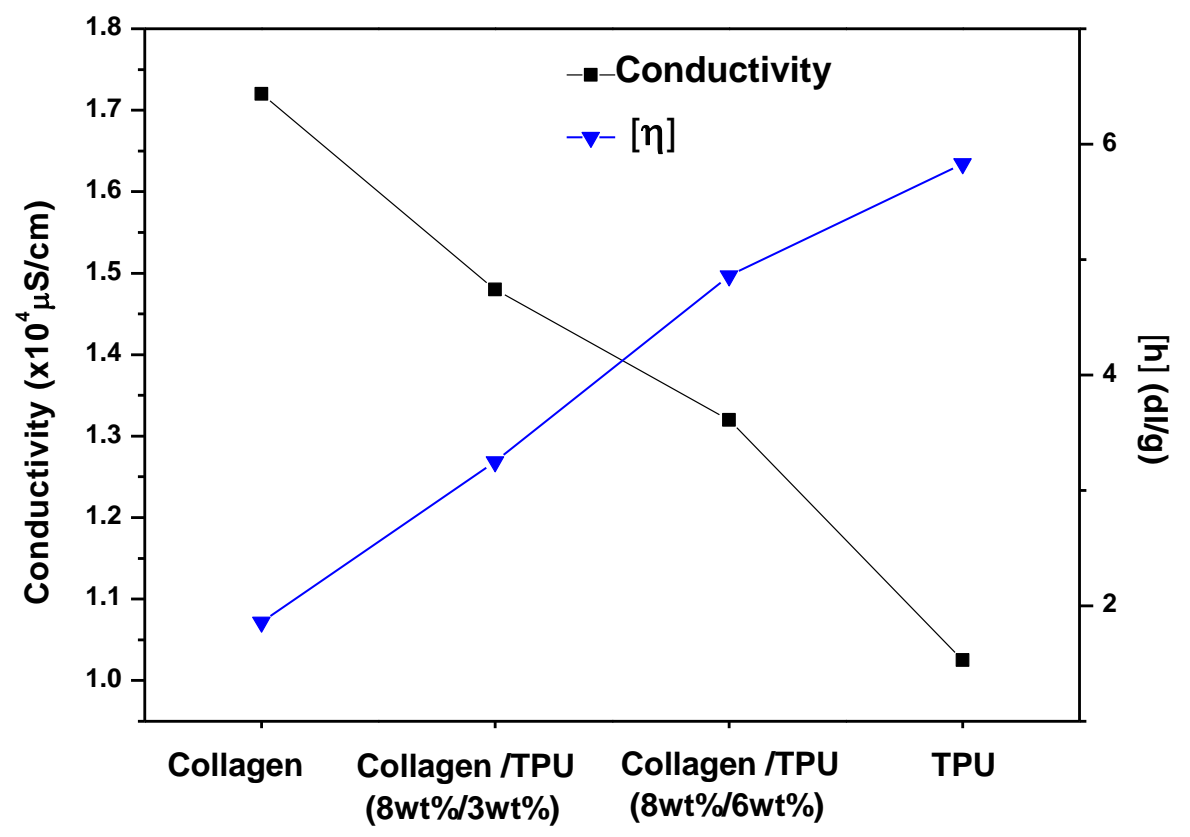

Fig. 2. The conductivity and [ $\eta]$ as the function of the collagen, TPU and collagen (shell)-TPU (core) complex solutions.

Tab. 1. Numerical statement of average diameters and standard deviation $(n=100)$.

\begin{tabular}{ccccc}
\hline Samples & TPU & $\begin{array}{c}\text { Collagen } \\
(\text { shell)/TPU (core) } \\
(8 \mathrm{wt} \% / 3 \mathrm{wt} \%)\end{array}$ & $\begin{array}{c}\text { Collagen } \\
(\text { shell)/TPU (core) } \\
(8 \mathrm{wt} \% / 6 \mathrm{wt} \%)\end{array}$ & Collagen \\
\hline $\begin{array}{c}\text { Average } \\
\text { Diameter }(\mu \mathrm{m}) \\
\begin{array}{c}\text { Stand } \\
\text { Deviation }(\mu \mathrm{m})\end{array}\end{array}$ & 0.289 & 0.72 & 0.96 & 0.15 \\
\hline
\end{tabular}

Fig. 3 showed the transmission electronspun micrographs of the shell-core structure of the different composite ultrafine fibers. The sharp core-shell interfaces with core phase TPU polymer encased by collagen shells were exhibited. This implied that the coaxial electrospinning was fast enough to prevent mixing of the core fluid with shell fluid. It was also found that some fibers displayed only monolayered structures instead of the core-shell structure. This could be attributed to the fact that the core fluid jet out of the coaxial capillaries might be split into a number of sub-jets during the electrospinning process $[10,11]$. So from Fig. 3 we could observe that overall fiber diameter was not uniform no matter how much concentration was used in core concentration. 


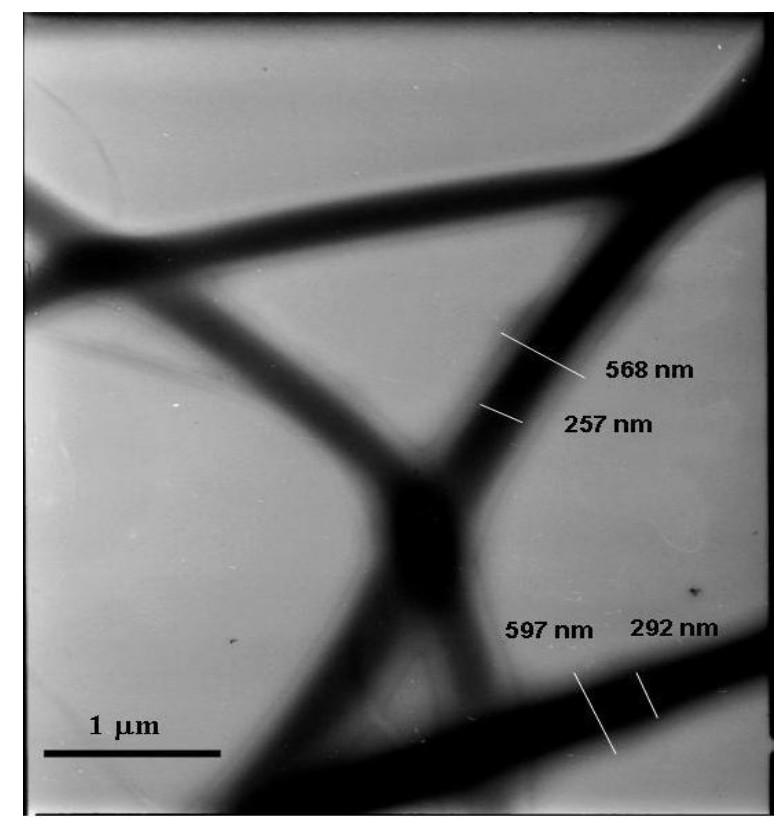

Fig. 3. Transmission electron microscopy image of the collagen (shell) - TPU (core) ultrafine fibers.

\section{Mechanical properties of TPU-collagen coaxial nanofibers}

The mechanical properties of core-shell structure nanofibers are important for their successful applications in tissue engineering. Collagen, TPU and their compound nanofibers were electrospun into $0.5 \mathrm{~mm}$ thick fiber mats to measure their mechanical properties. Fig. 4 showed the typical stress-strain curve of collagen/TPU coaxial electrospun nanofibrous mats and pure collagen and TPU under tensile loading. The electrospun TPU material gave a characteristic response for elastomeric materials-sigmoidal in shape. It showed a very soft and flexible characteristic with low Young's modulus and the high elongation at break of $300 \%$. In contrast, the electrospun collagen materials exhibited plastic mechanical properties with high initial modulus and poor elongation at break of $15 \%$. From the stress-strain curves of collagen/TPU coaxial electrospun mats, we could observe that with increasing the concentration of core TPU solutions, the strain of the materials became larger and the initial modulus became lower. Therefore, we could adjust the mechanical property to meet the requirement in practice through changing the TPU concentration in the coaxial system.

To design an ideal scaffold, various factors should be considered, such as pore size and morphology, mechanical properties versus porosity, surface properties and appropriate biodegradability. Of these factors, the importance of mechanical properties on cell growth is particularly obvious in tissues, such as bone, cartilage, blood vessels, tendons, heart valve and muscles. Different natural tissues have different mechanical properties. TPU had high tensile strength, good tear and abrasion resistance. But its Young's modulus was very low. Collagen had high Young's modulus and excellent biocompatibility, but its tensile strength and strain were poor. In order to mimic the mechanical properties in the radial and circuit direction, we need to adjust the coaxial-electrospinning process parameter. From the analysis, we could find that with the TPU concentration ingredient increasing, the Young's modulus, tensile strength and tensile strain changed. Coaxial nanofibers may have improved mechanical strength compared with pure non-crosslinked 
collagen nanofibers, which could combine the advantages of both natural and synthetic materials. The tensile strength and ultimate strain obtained from four independent tests are summarized in Table 2.

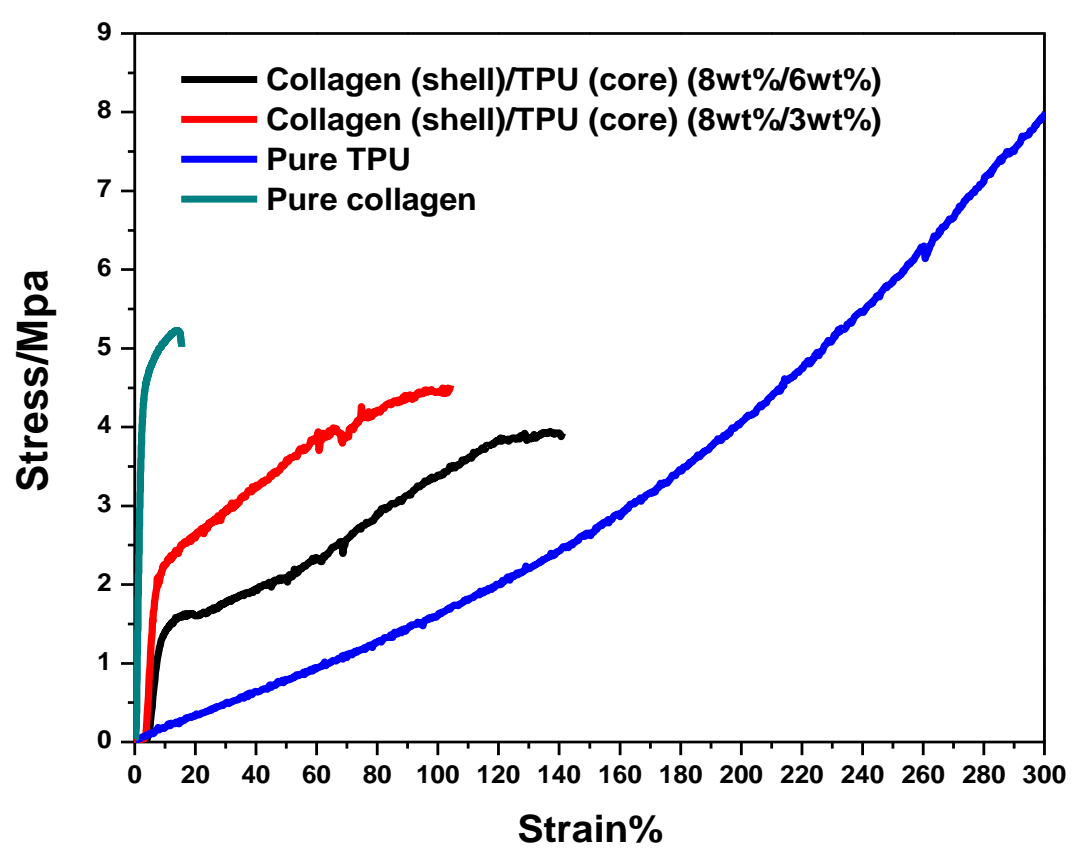

Fig. 4. Typical stress-strain curve for the electrospun TPU, collagen and PU/collagen Coaxial compound nanofibers.

Tab. 2. Mechanical tensile properties of collagen/TPU coaxial nanofibrous scaffolds, $(\mathrm{n}=4)$.

\begin{tabular}{ccccc}
\hline Samples & $\begin{array}{c}\text { Pure } \\
\text { TPU }\end{array}$ & $\begin{array}{c}\text { Pure } \\
\text { collagen }\end{array}$ & $\begin{array}{c}\text { Collagen } \\
\text { (shell)/TPU } \\
\text { (core) }(8 \mathrm{wt} \% / 3 \\
\mathrm{wt} \%)\end{array}$ & $\begin{array}{c}\text { Collagen } \\
\text { (shell)/TPU } \\
(\text { core })(8 \mathrm{wt} \% / 6 \\
\mathrm{wt} \%)\end{array}$ \\
\hline $\begin{array}{c}\text { Tensile } \\
\text { stress(MPa) }\end{array}$ & $7.7 \pm 0.7$ & $4.8 \pm 0.7$ & $4.53 \pm 0.3$ & $3.9 \pm 0.24$ \\
$\begin{array}{c}\text { Ultimate stain } \\
(\%)\end{array}$ & $365 \pm 24.5$ & $13.7 \pm 1.9$ & $142.8 \pm 7.2$ & $145.6 \pm 4.1$ \\
\hline
\end{tabular}

\section{Conclusions}

In this study, the HFP was found as an appropriate solution for collagen/TPU coaxial electrospinning and this compound core-shell structured nanofiber was prepared for the first time in our lab. The morphology and micro-structure of the resulting composite nanofibers were characterized through SEM and TEM. The diameters of spun nanofibers were influenced by core concentration of TPU solution and the proper core solution was one important parameter affecting the fiber morphology in the shell-core structure. The mechanical behavior of the nanofibers membranes was also investigated and the collagen/TPU coaxial complex represented a potential ideal 
tissue engineering scaffold and a promising functional biomaterial. Further investigation is going on to explore the application of coaxial electrospun collagen/TPU complex in tissue engineering.

\section{Experimental}

\section{Materials}

The polymer of thermoplastic polyurethane (Tecoflex EG-80A) was purchased from Noveon, Inc. (USA) and collagen (mol.wt, $0.8-1 \times 10^{5} \mathrm{Da}$ ) was purchased from Sichuan Ming-rang Bio-Tech Co. Ltd (China). The solvent used 1,1,1,3,3,3,-hexafluoro-2propanol (HFP) was brought from Daikin Industries Ltd (Japan).

\section{Scaffold fabrication by coaxial electrospinning}

In our experiment, different solvents were used to investigate the formation of an appropriate Taylor cone in the process of coaxial electrospinning. In this study, collagen and TPU were both dissolved in HFP solvent separately. The collagen solution was made in a weight ratio of $8 \mathrm{wt} \%$ and TPU solutions were made with different concentrations which varied from $3 \%$ to $6 \%$. The prepared solutions were resolved using multipoint heating magnetic stirring apparatus with sufficient stirring at room temperature.

The basic experimental schematic illustration used was the coaxial electrospinning instrument. Briefly, it consisted of syringe-like apparatus with an inner needle coaxially placed inside an outer one. Two immiscible liquids were injected at appropriate flow rate through this coaxially steel needles arranged. Both needles were connected to the same electrical potential. A high electrospinning voltage was applied between the needle and ground collector using a high voltage power supply. The electric field generated by the surface charge caused the solution drop at the tip of the needle to distort into a Taylor cone. With an increase in the supplied high voltage to a threshold value, a steady coaxial compound fluid jet with an out meniscus surrounding the inner one was formed and ejected out of the Taylor cone. The fluid jet was then thinning into sub-micrometer scale as a consequence of bending instability. After evaporation of the solvents during the course of flying, the thin jet was deposited on the collector and resulted in a bi-component composite fibrous membrane.

In this experiment, the outer needle and the inner needle were connected through two silicones tube to syringes which contained the shell solution and core solution. A high voltage DC power supply (BGG6-358, BMEICO.LTD, China) that generated 20 $\mathrm{KV}$ in the present work and a grounded metallic screen to collect the ultrafine fibers. The distance between the spinneret and the collector was $130-150 \mathrm{~cm}$. The electrospinning was done at ambient temperature with humidity of $55 \%$. The core and shell flow rates were $0.8 \mathrm{ml} / \mathrm{h}$ and $1.2 \mathrm{ml} / \mathrm{h}$ respectively and the resulting nonwoven fibrous mats had a thickness of around $0.10-0.20 \mathrm{~mm}$ and then they were placed into a vacuum oven for 24 hours to remove residual solvent.

\section{Characterization}

The morphologies and diameters of the nanofibers electrospunned by pure and coaxial of TPU to collagen were determined with SEM (JEOL, JSM-5600, Japan) at a accelerated voltage of $15 \mathrm{KV}$. Verification of core-shell structure was calculated by 
TEM ( $\mathrm{H}-800$, Hitachi) at $100 \mathrm{kV}$, and the samples for TEM observations were prepared by collecting the nanofibers onto carbon-coated $\mathrm{Cu}$ grids. The diameter range of the fabricated nanofibers was measured based on the SEM images using image visualization software Image J $1.34 \mathrm{~s}$ (National Institutes of Health, USA). Average diameter and diameter distribution were determined by measuring one hundred random nanofibers from SEM images. Conductivity of TPU, collagen and TPU/collagen blend solution were measured by electric conductivity meter. The intrinsic viscosity ([n]) of the solution was measured by using Ubbelohde viscometer. Mechanical measurements were carried out by applying tensile test loads to samples which were prepared from electrospun ultra fine non-woven fiber mats.

\section{Acknowledgements}

This research was supported by National science foundation (30570503), National high technology research and developed program (863 Program, 2008AA03Z305), Science and technology commission of Shanghai municipality program (08520704600, and 0852nm03400).

\section{References}

[1] Zhang, Y, Z.; Venugopal, J.; Huang, Z. M.; Lim, C. T.; Ramakrishna, S. Biomacromolecules 2005, 6, 2583.

[2] Langer, R.; Vacanti, J. P. Science 1993, 260, 920.

[3] Weinand, C.; Pomerantseva, I., Neville, C. M.; Gupta, R.; Weinberg, E.; Madisch, I.; Shapiro, F.; Abukawa, H.; Troulis, M. J.; Vacanti, J. P. Bone 2006, 38, 555.

[4] Nair, L. S.; Bhattacharyya, S.; Laurencin, C. T. Expert Opin Biol Ther 2004, 4, 659.

[5] Matthews, J. A.; Wnek, G. E.; Simpson, D. G.; Bowlin, G. L. Biomacromolecules 2003, 3, 232.

[6] Demir, M. M.; Yilgor, I.; Yilgor, E.; Eman, B. Polymer 2002, 43, 3303.

[7] Pham, Q. P.; Shama, U.; Mikos, A. G.; Tissue Eng 2006, 12, 1197.

[8] Heydarkhan-Hagvall, S.; Schenke-Layland, K.; Dhanasopon, A. P.; Rofail, F.; Smith, H.; Wu, B. M.; Shemin, R.; Beygui, R. E.; Maclellan, W. R. Biomaterials 2008, 29, 2907.

[9] Yagi, K.; Michibayashi, N.; Kurikawa, N.; Nakashima, Y.; Mizoguchi, T.; Harada, A.; Higashiyama, S.; Muranka, H.; Kawase, M. Biol Pharm Bull 1997, 20, 1290.

[10] He, C. L.; Huang, Z. M.; Han, X. J.; Liu, L.; Zhang, H. S.; Chen, L. S. J Macromol Sci Phys 2006, 45, 515.

[11] Huang Z. M.; He, C. L.; Yang, A. Z.; Zhang, Y. Z.; Han, X. J.; Yin, L.; Wu, Q. S. J. Biomed Mater Res 2006, 77A, 169. 